\title{
Dimorfismo em machos de Euglossa mandibularis Friese (Apidae, Euglossina)
}

\section{Rui Carlos Peruquetti ${ }^{1}$}

\begin{abstract}
Male dimorphism in Euglossa mandibularis Friese (Apidae, Euglossina). Males of Euglossa mandibularis Friese, 1899 can be categorized by (1) presence or (2) absence of scutellar tuft (a morphological feature of dense setae found in females' scutellum of the genera Euglossa Latreille, 1802 and Eulaema Lepeletier, 1841). A multivariate statistical analysis using six morphological measures (hindtibia length and width, head width, intertegular span, midbasitarsus length and scape length) show that males of the categories 1 and 2 were significantly different in relation to such measures. The width head, hindtibia length and scape length were the measures that most influenced the observed differences. Levene test suggest there is not effect of stabilizing selection on males without scutellar tuft. Since there is a fluctuation in the proportion of males with scutellar tuft occurring in a single population, the observed morphological differences might be environmentally determined.

KEY WORDS. Apidae, Euglossini, Euglossa, male dimorphism
\end{abstract}

Euglossa mandibularis Friese, 1899 é relativamente comum em áreas de Mata Atlântica (MOURE 1995), onde seus machos são importantes polinizadores de algumas espécies de Cyphomandra (Solanaceae) (SOARES et al. 1989). Estes machos podem ser separados em machos com tufo escutelar (MCT; característica presente em fêmeas de Euglossa Latreille, 1802 e Eulaema Lepeletier, 1841; MOURE 1967; KIMSEY 1987) e machos sem tufo escutelar (MST).

Este trabalho teve como objetivo (1) verificar a possível existência de outras diferenças morfológicas entre estes dois tipos de machos; (2)'testar a hipótese de que os MST teriam um tamanho ótimo, isto é, sofreriam ação de seleção estabilizadora e entre eles haveria menos variabilidade morfológica do que entre os MCT. Até o momento, conhece-se MCT apenas em E. mandibularis e assumiu-se que estes poderiam não ter sucesso reprodutivo por apresentarem impedimentos morfológicos e/ou genéticos associados à presença do tufo escutelar. Paka testar estas hipóteses foram tomadas seis medidas (comprimento e largura da tíbia do terceiro par de pernas, largura da cabeça, distância intertegular, comprimento do basitarso do segundo par de pernas e comprimento do escapo) em 19 MCT e em 17 MST (Tab. I). Estas medidas foram escolhidas por serem de fácil determinação, refletirem o peso dos indivíduos (largura da cabeça e distância intertegular) (CANE 1987; RUST 1991; BULLOCK 1999) e estarem em estruturas envolvidas no processo de coleta de compostos aromáticos (antena, basitarso do segundo par de pernas e tíbia do terceiro

1) Laboratório de Genética Bioquímica, Departamento de Genética e Evolução, Universidade Federal de São Carlos. Caixa Postal 676, 13567-905 São Carlos, São Paulo, Brasil.

E-mail: prcp@iris.ufscar.br 
par de pernas) (EVOY \& JONES 1971; KIMSEY 1984). Todos os 36 machos analisados foram amostrados em Viçosa (Minas Gerais, 2045'14'S, 4252'55"W) quando coletavam compostos aromáticos em flores de Cyphomandra calycina Sendtn (SOARES et al. 1989). Fragrâncias aromáticas fazem parte da biologia reprodutiva de Euglossina, mas a maneira como elas são empregadas pelos machos ainda não é totalmente compreendida (ElTZ et al. 1999; PERUQueTti 2000). Para testar a hipótese (1) as medidas foram analizadas conjuntamente através do teste de Hotelling e análise de componentes principas; a hipótese (2) foi testada utilizando-se o teste de Levene para homogeneidade de variância. Estes procedimentos estatísticos estão detalhados em MANLY (1994).

Tabela I. Medidas tomadas em machos de Euglossa mandibularis com tufo escutelar (MCT) e sem tufo escutelar (MST). (CT) Comprimento da tíbia do terceiro par de pernas, (LT) largura da tíbia do terceiro par de pernas, (LC) largura da cabeça, (DIT) distância intertegular, (CBT) comprimento do basitarso do segundo par de pernas, (CE) comprimento do escapo. As medidas são dadas em milímetros $\left(\bar{x}=\right.$ média, $s^{2}=$ variância). Entre parênteses o número de machos utilizados. Os MCT diferem significativamente dos MST (Teste de Hotelling, $T^{2} ; F(6,29)$ $=3,30 ; p<0,05)$.

\begin{tabular}{lccccc}
\hline \multirow{2}{*}{ Medidas } & \multicolumn{2}{c}{ MCT $(n=19)$} & & \multicolumn{2}{c}{ MST $(n=17)$} \\
\cline { 2 - 3 } \cline { 5 - 6 } & $\bar{X}$ & $s^{2}$ & & $\bar{X}$ & $s^{2}$ \\
\hline CT & 5,57 & 0,112 & & 5,83 & 0,114 \\
LT & 3,46 & 0,073 & & 3,36 & 0,076 \\
LC & 5,48 & 0,083 & & 5,74 & 0,109 \\
DIT & 4,37 & 0,063 & & 4,61 & 0,058 \\
CBT & 2,70 & 0,031 & & 2,71 & 0,016 \\
CE & 1,21 & 0,011 & & 1,30 & 0,005 \\
\hline
\end{tabular}

Verificou-se que os MST e MCT diferem entre si em relação às medidas tomadas (teste de Hotelling, $\left.\mathrm{T}^{2} ; \mathrm{F}(6,29)=3,30 ; \mathrm{p}<0,05\right) \mathrm{e}$, de maneira geral, os MCT são menores do que os MST (Tab. I). O comprimento da tíbia do terceiro par de pernas, a largura da cabeça e o comprimento do escapo foram as medidas que mais influenciaram na diferença observada. Elas dominaram o primeiro componente da análise ( $42,8 \%$ da variação observada) (Tab. II).

O teste de Levene indica que, na população estudada, não há efeito de seleção estabilizadora sobre os MST ( $p>0,05$ para todas as medidas). Desta maneira, os MST apresentaram variabilidade morfológica semelhante aos MCT e não haveria entre os primeiros um tamanho ótimo. Ausência de tamanho "ótimo" também foi observada em machos com sucesso reprodutivo efetivo em vários grupos de Hymenoptera (AlCOCK 1995; MolumBy 1997).

Conhece-se pouco sobre a biologia de E. mandibularis. Entretanto, devido às flutuações das frequiências dos MCT na população estudada (em alguns anos $50 \%$ + dos machos amostrados apresentam tufo escutelar; observação pessoal), é possível sugerir que as diferenças observadas (inclusive a presença do tufo escutelar) 
seriam produto de influência ambiental durante o desenvolvimento dos machos. Como acontece em outras abelhas cujos machos são dimórficos em relação a tamanho do corpo e ausência/presença de certas estruturas (DANFORTH \& NEFF 1992; KUKUK 1996).

Tabela II. Valores da análise de componentes principais (PCA) com seis medidas tomadas em machos de Euglossa mandibularis com e sem tufo escutelar $(n=36)$. O teste de Levene sugere que não há efeito de seleção estabilizadora sobre os machos sem tufo da população estudada ( $p>0,05$ para todas as medidas). (CT) Comprimento da tíbia do terceiro par de pernas, (LT) largura da tíbia do terceiro par de pernas, (LC) largura da cabeça, (DIT) distância intertegular, (CBT) comprimento do basitarso do segundo par de pernas, (CE) comprimento do escapo.

\begin{tabular}{lcc}
\hline Medidas & Componente 1 & Componente 2 \\
\hline CT ${ }^{*}$ & $0,75^{\star \star}$ & 0,15 \\
LT & $0,17^{\star \star}$ & $-0,88^{\star \star}$ \\
LC & $0,78^{\star \star}$ & 0,05 \\
DIT & 0,64 & $-0,40$ \\
CBT & 0,51 & 0,32 \\
CE & $0,84^{\star \star}$ & 0,01 \\
\hline Variação (\%) & 42,80 & 17,80 \\
\hline
\end{tabular}

$\left(^{*}\right)$ A tíbia do terceiro par de pernas difere entre os dois tipos de machos em seu tamanho (primeiro componente da PCA) e forma (segundo componente da PCA); $\left(^{* *}\right)$ indica os valores que mais contribuíram $(>0,70)$ para as diferenças morfométricas observadas entre os dois tipos de machos.

Apesar da falta de evidências, é possível supor que os MCT, além de coletarem compostos aromáticos como os MST, sejam capazes de reproduzir-se e a presença do tufo escutelar não estaria relacionada à diplóidia nestes machos. Em Hymenoptera, machos diplóides são inviáveis e, normalmente, não se reproduzem (COOK 1993).

Entre 51 machos de E. mandibularis amostrados em Viçosa e analisados por TAKAHASHI et al. (2001), apenas um foi diplóide. Esta freqüência observada está de acordo com a esperada para as populações de himenópteros arrenótocos panmíticos (até 10\%). Entretanto, a freqüência esperada estimada de machos diplóides (OWEN \& PACKER 1994) nesta população é de $20,6 \% \pm 20,4$ (DP), o que pode ser uma superestimativa, dado o tamanho da amostra e as frequêencias alélicas observadas no sistema enzimático (malato desidrogenase) no qual foi detectado o macho diplóide.

Desta forma, aparentemente, a presença dos MCT não contribui significativamente para a redução do tamanho efetivo da população estudada. Caso isto acontecesse, por razões genéticas ou morfológicas, freqüências de 50\% de MCT, por exemplo, reduziria de imediato o tamanho efetivo da população amostrada à metade (desde que todos os MST tenham a mesma chance de acasalamento), o que favoreceria o acasalamento entre irmãos e irmãs e elevaria a frequiência de machos diplóides na população amostrada a níveis bem maiores do que os apresentados (PACKER \& OWEN 2001). Porém, estudos genéticos comparando MCT e MST podem reforçar esta hipótese. 
AGRADECIMENTOS. Gostaria de agradecer ao Sr. Geraldo Paiva pelo auxílio no trabalho de campo. À Patrícia Santos Ferreira-Peruquetti e Daniele Boraschi pela leitura da primeira versão do manuscrito e pelas sugestões apresentadas. Aos consultores anônimos da RBZ pelas sugestões que contribuíram muito para a melhoria do trabalho. Este trabalho foi financiado em parte pela FAPEMIG. A FAPESP contribuiu com o auxílio financeiro (Proc. 98/16390-0) durante a redação do manuscrito.

\section{REFERÊNCIAS BIBLIOGRÁFICAS}

ALCOCK, J. 1995. Body size and its effect on male-male competition in Hylaeus alcyoneus (Hymenoptera: Colletidae). Jour. Insect Behav. 8 (2): 149-159.

BuLLOCK, S.H. 1999. Relationships among body size, wing size and mass in bees form a tropical dry forest in México. Jour. Kansas Entomol. Soc. 72 (4): 426-439.

CANE, J.H. 1987. Estimation of bee size using intertegular span (Apoidea). Jour. Kans. Entomol. Soc. 60 (1): 145-147.

CooK, J.M. 1993. Sex determination in the Hymenoptera: a review of models and evidence. Heredity 71: 421-435.

DANFORTH, B.N. \& J.L. NeFF. 1992. Male polymorphism and polyethism in Perdita texana (Hymenoptera: Andrenidae). Ann. Ent. Soc. Amer. 85 (5): 616-626.

Eltz, T.; W.M. WhitTen; D.W. RoubiK \& K.E. LinsenMair. 1999. Fragrance collection, storage, and accumulation by individual male orchid bees. Jour. Chem. Ecol. 25 (1): 157-176.

EvoY, W.E. \& B.P. JoNEs. 1971. Motor patterns of males euglossine bees evoked by floral fragrances. Anim. Behav. 19: 583-588.

KIMSEY, L.S. 1984. The behavioural and structural aspects of grooming and related activities in euglossine bees (Hymenoptera). Jour. Zool., London, 204: 541-550.

. 1987. Generic relationships within the Euglossini (Hymenoptera: Apidae). Syst. Entomol. 12: 63-72.

KUKUK, P.F. 1996. Male dimorphism in Lasioglossum (Chialictus) hemichalceum: the role of larval nutrition. Jour. Kans. Entomol. Soc. 69 (4 suppl.): 147-157.

MANLY, B.F.J. 1994. Multivariate statistical methods. A primer. London, Chapman \& Hall, $2^{\text {nd }}$ ed., $215 \mathrm{p}$.

Molumby, A. 1997. Why make daughter larger? Maternal sex-allocation and sex-dependent selection for body size in a mass-provisioning wasp, Trypoxylon politum. Behav. Ecol. 8 (3): 279-287.

Moure, J.S. 1967. A checklist of the known euglossine bees. Simp. Biota Amazônica, Manaus, 5: 395-415.

1995. Notas sobre algumas espécies de abelhas da Bahia, Brasil (Hymenoptera, Apoidea). Revta bras. Zool. 12 (3): 467-470.

OWEN, R.E. \& L. PACKer. 1994. Estimation of the proportion of diploid males in populations of Hymenoptera. Heredity 72: 219-227.

PACKer, L. \& R. OWen. 2001. Population genetic aspects of pollinator decline. Conserv. Ecol. 5 (1): (4) [On line] URL: http://www.consecol.org/Vol5/iss1/art4.

PERUQUeTti, R.C. 2000. Function of fragrances collected by Euglossini males (Hymenoptera: Apidae). Entomol. Gener. 25 (1): 33-37.

Rust, R.W. 1991. Size-weight relationship in Osmia lignaria propinqua Cresson (Hymenoptera: Megachilidae). Jour. Kans. Entomol. Soc. 64 (2): 174-178.

Soares, A.A.; L.A.O. Campos; M.F. Vieira \& G.A.R. Melo. 1989. Relações entre Euglossa (Euglossella) mandibulares Friese, 1899 (Hymenoptera, Apidae, Euglossini) e Cyphomandra calycina (Solanaceae). Ciên. Cult. 41 (9): 903-905.

TAkahashi, N.C.; R.C. Peruquetti; M.A. Del Lama \& L.A.O. Campos. 2001. A reanalysis of diploid male frequencies in euglossine bees (Hymenoptera: Apidae). Evolution 55 (9): 1897-1899.

Recebido em 13.V.2002; aceito em 29.X.2002. 